\title{
La estimulación eléctrica transcutánea de tipo acupuntura como herramienta aceleradora de la recuperación de los tejidos expuestos a sobrecarga vocal
}

\author{
Christopher Fuentes Aracena ${ }^{1}$; Javiera Arrau Bordalí²; Gabriela Pino Quiroz²; Nataly Valdivia Alarcón²; \\ Camila Aguilar Pavez ${ }^{2}$
}

Recibido 25 de junio de 2019 / Revisado7 de octubre de 2019 / Aceptado 25 de noviembre de 2019

Resumen. Tradicionalmente, se ha planteado que el reposo es la herramienta ideal para permitir la recuperación natural de los tejidos inducidos a sobrecarga. Sin embargo, los tiempos necesarios para que esto se produzca son muy largos. Se ha estimado que la electroterapia es útil al momento de recuperar algunos tejidos sobreutilizados, no obstante, se desconocen estos beneficios en la función vocal. Este estudio tuvo como objetivo describir los efectos de la electroterapia sobre los tejidos laríngeos y cordales que previamente fueron sometidos a sobrecarga vocal. En esta investigación participaron 21 mujeres sin patología o entrenamiento vocal, cuya edad fue de $24 \pm 1,28$ años. Las participantes fueron sometidas a sobrecarga vocal por 40 minutos y posteriormente, a 15 minutos de estimulación eléctrica transcutánea (TENS) de tipo acupuntura. A través de laringoscopía con luz continua, análisis acústico-perceptual y estudio sintomatológico, se examinaron los cambios provocados por la electroterapia. Los principales resultados incluyen a la relajación perilaríngea y de los músculos intrínsecos sobreutilizados, y eliminación de los síntomas sufridos. De esta forma, se concluye que la TENS de tipo acupuntura es una herramienta útil en la aceleración de la recuperación de los tejidos sometidos a sobrecarga vocal.

Palabras claves: carga vocal; electroterapia; fatiga vocal; hiperfunción vocal; TENS.

\section{[en] Acupuncture-like transcutaneous electrical nerve stimulation as an accelerating tool for the recovery of the tissues exposed to vocal overload.}

\begin{abstract}
Traditionally, it has been suggested that rest is the ideal tool to allow natural recovery of tissues induced by overload. However, the times necessary for this to occur are very long.

It has been estimated that electrotherapy is useful when it comes to recover some overused tissues, however, these benefits in vocal function are unknown. The purpose of this study was to describe the effects of acupuncture-like transcutaneous electrical nerve stimulation on the laryngeal and cordal tissues that were previously subjected to vocal overload. In this research, 21 women without pathology or vocal training participated, whose age was $24 \pm 1.28$ years. The participants were subjected to vocal overload for 40 minutes and subsequently to 15 minutes of acupuncture-like transcutaneous electrical nerve stimulation.

Through continuous laryngoscopy, acoustic-perceptual analysis and symptomatic study, the changes caused by electrotherapy were examined. The main results include perilaryngeal and intrinsic laryngeal muscles relaxation, and elimination of the symptoms suffered. This way it is concluded that electrostimulation is a useful tool in the acceleration of tissue recovery subjected to vocal overload.

Key words: electrotherapy, TENS; vocal fatigue; vocal hyperfunction; vocal load.

Sumario: 1. Introducción. 2. Objetivo. 3. Metodología. 3.1. Diseño. 3.2. Participantes. 3.3. Instrumentos. 3.4. Procedimiento. 4. Resultados. 4.1. Resultados previos a la tarea de sobrecarga. 4.2. Resultados posteriores a la tarea de sobrecarga (previo a la electroestimulación). 4.3. Resultados posteriores a la electroterapia. 5. Discusión. 5.1 Efectos sobre la región perilaríngea. 5.2. Efectos sobre los músculos intrínsecos. 5.3. Efectos sobre la cubierta cordal. 5.4. Efectos del reposo vocal posterior a la sobrecarga vocal. 6. Conclusiones. 7. Bibliografía.
\end{abstract}

Cómo citar: Fuentes Aracena, C. et al. (2020) La estimulación eléctrica transcutánea de tipo acupuntura como herramienta aceleradora de la recuperación de los tejidos expuestos a sobrecarga vocal, Revista de investigación en Logopedia 10(1), 31-41.

\footnotetext{
Facultad de Ciencias de la rehabilitación, Universidad Andrés Bello, Santiago, Chile. chrfuentes@gmail.com

Facultad de Ciencias de la rehabilitación, Universidad Andrés Bello, Santiago, Chile. 


\section{Introducción}

La sobrecarga es parte del continuo de carga vocal y se relaciona con la aparición de signos o síntomas que expresan la exigencia de la tarea (Peterson, Pistilli, Haff, Hoffman, \& Gordon, 2011). Su acumulación en el tiempo favorece el surgimiento de daños estructurales de mayor cuantía, mientras que su adecuada prescripción, permite la adquisición de nuevos rendimientos (Phillips \& Kennedy, 2012). En la función vocal las injurias que provoca se conocen como trastornos de base funcional, e incluyen a expresiones como pólipos, pseudoquistes, entre otros (Bastian \& Thomas, 2016). No obstante, a nivel perilaríngeo también existen manifestaciones que nacen de la aplicación de constantes y excesivas fuerzas mecánicas sobre los ligamentos, músculos o tendones que rodean a la laringe y hioides (O’Rourke, Attique, Rehman, Saunders, \& Fenton, 2014). De esta forma, se ha documentado la aparición de tendinopatías (Aydil, Ekinci, Köybaşioğlu, \& Kizil, 2007), rupturas ligamentosas (Stern, Jackson-Menaldi, \& Rubin, 2013) y alteraciones miofasciales (Jung et al., 2017).

Uno de los puntos en común que tienen las lesiones que se producen por sobrecarga es la inflamación, la que con el tiempo, se vuelve crónica y expresa cuadros como los anteriormente comentados (Bisciotti \& Eirale, 2013). Tradicionalmente, se ha planteado que el descanso o reposo es la forma más efectiva para realizar la recuperación y, con esto, disminuir la opción de cronicidad de la inflamación (Whitling, Lyberg-Åhlander, \& Rydell, 2017). Sin embargo, sus efectos sobre los tejidos laríngeos y cordales son lentos, y tienden a ser incompatibles con el uso diario que requieren algunos profesionales de la voz (Rousseau et al., 2011). Se ha visto que con el reposo los tiempos de recuperación pueden llegar hasta las 24 horas posterior a la sobrecarga vocal (Whitling et al., 2017). Mientras que en otros trabajos, se ha indicado que dos horas de reposo bastarían, principalmente, para disminuir la fatiga de los músculos intrínsecos de la laringe (McCabe \& Titze, 2002).

Debido a los lentos resultados que suele entregar el reposo vocal, se han buscado una serie de alternativas que permitan disminuir la cronicidad de la inflamación y, por consiguiente, interceptar el daño que produce la sobrecarga vocal. Desde el estudio de los pliegues vocales, se ha indicado que el uso del estrés tensil es capaz de atenuar el proceso inflamatorio existente en su cubierta (Verdolini Abbott et al., 2012). También se han publicado casos clínicos, donde con 45 minutos de ejercicios que aumenten la tensión de la cubierta cordal, se ha reducido notoriamente su inflamación (Fuentes, 2018).

Desde la fisioterapia, se han presentado una serie de agentes físicos que permiten la recuperación muscular, como la electroterapia (Babault, Cometti, Maffiuletti, \& Deley, 2011), la termoterapia o la crioterapia (Petrofsky et al., 2015).

En la clínica vocal, el agente físico que se usa con mayor frecuencia es la electroterapia (de Oliveira et al., 2015), tanto en su modalidad TENS (estimulación nerviosa eléctrica transcutánea), como EMS (estimulación muscular funcional). Se ha planteado que dentro de estas modalidades la que mejor resultado entrega al momento de recuperar los tejidos expuestos a sobrecarga vocal es la EMS (Fowler, Awan, Gorham-Rowan, \& Morris, 2011), ya que con su aplicación se genera la estimulación muscular necesaria para aumentar el flujo sanguíneo y así, acelerar la eliminación de desechos metabólicos. Sin embargo, también se ha observado que a pesar del beneficio anteriormente mencionado, los efectos secundarios incluyen la aparición de dolor perilaríngeo o el aumento de tensión laríngea (Fowler et al., 2011).

Se han presentado dos grades modalidades de corriente TENS, una que utiliza frecuencias en el orden de los 50 a $150 \mathrm{~Hz}$ (de tipo convencional) y otra cuyas frecuencias se encuentran entre 1 a $4 \mathrm{~Hz}$ (de tipo acupuntura o de baja frecuencia) (Cameron, 2014). En la terapéutica vocal, tienden a utilizarse con mayor periodicidad aquellas que fluctúan entre los 10 (Guirro et al., 2008) y $100 \mathrm{~Hz}$ (Mansuri et al., 2019).

Desde la fisioterapia, se han observado beneficios que permiten comprender el potencial terapéutico de la TENS de tipo acupuntura (de baja frecuencia). Dentro de estos se encuentran la reducción del dolor y analgesia ocasionada por espasmos o contracturas musculares (Jun, Kim, \& Kim, 2015), disminución de la actividad electromiográfica de los músculos del aparato masticatorio y por consiguiente, descenso del dolor originado de su disfunción (Esclassan et al., 2017; Mummolo et al., 2018). Además, debido a las vibraciones mecánicas que ofrece durante su uso (Hansson \& Ekblom, 1983), permite una sensación de analgesia y relajación, en tiempo y cantidad, superior a la TENS de tipo convencional (Tousignant-Laflamme et al., 2013). Por último, favorece la recuperación de los tejidos fatigados con el ejercicio, gracias a que promueve la eliminación de desechos metabólicos (Kang, Jeon, \& Lee, 2015).

A pesar de los múltiples beneficios que ofrece el uso de TENS de baja frecuencia, en la intercepción y rehabilitación de la patología vocal este no ha sido explorado, por lo que sus reales repercusiones se desconocen.

\section{Objetivo}

Describir los efectos de la estimulación nerviosa eléctrica transcutánea de tipo acupuntura sobre los tejidos laríngeos y cordales que previamente fueron sometidos a sobrecarga vocal. 


\section{Metodología}

\subsection{Diseño}

Corresponde a un estudio de diseño experimental y cuantitativo, de carácter descriptivo.

\subsection{Participantes}

Para este estudio se consideraron a 21 mujeres sin entrenamiento o patología vocal. La edad de las participantes fue de $24 \pm 1,28$ años.

\subsection{Instrumentos}

La grabación de la voz de cada participante se realizó con un micrófono Shure sm58, el que estuvo sostenido con un atril que evitó su desplazamiento. La señal fue procesada por la interface Audiobox USB, la cual estaba conectada a un notebook Toshiba L845. Las grabaciones se llevaron a cabo con el software Audacity 2.3.0, con una frecuencia de muestreo de $44.000 \mathrm{~Hz}$ y 16 bit de cuantificación.

La intensidad inicial, la utilizada durante la tarea de sobrecarga y la conseguida después de la electroestimulación, fue obtenida gracias a un sonómetro de marca Benetech - GM1356. Durante la tarea de sobrecarga vocal este mismo instrumento se conectó a un atril para controlar la intensidad vocal de la participante.

Para observar el comportamiento supraglótico y la coloración cordal y perilaríngea, se utilizó un laringoscopio de marca Ecleris

La electroterapia se llevó a cabo gracias a la utilización de un equipo portátil de marca y modelo Care Tec IV. Para permitir la conducción del impulso eléctrico se utilizó gel de marca DifemPharma. Se usaron cuatro electrodos de goma, los que se limpiaron con alcohol posterior a cada aplicación. Sus dimensiones eran de $3 \times 2 \mathrm{~cm}$ para los utilizados en la región submandibular y $5 \times 3 \mathrm{~cm}$ para los colocados en ambos costados del cartílago tiroides (figura 1). Para asegurar su fijación se utilizó cinta micropore de marca 3M (figura 1).

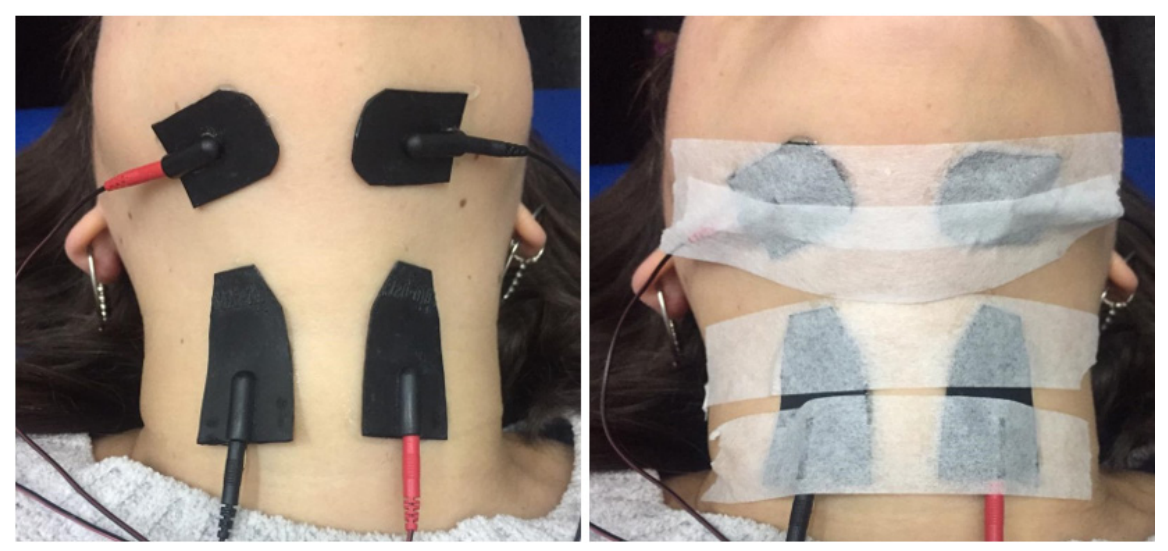

Figura 1. Posición y fijación de los electrodos.

\subsection{Procedimiento}

Para llevar a cabo este estudio experimental se realizaron una serie de procedimientos que permitieron obtener datos previos a la tarea de sobrecarga y, posteriormente, antes y después del uso de electroterapia.

Antes de la tarea de sobrecarga se grabó la voz de cada participante. Se le solicitó a cada una de ellas que prolongara una vocal /a/ por el mayor tiempo posible. Esta emisión se debió realizar con una voz habitual en lo que respecta a tono e intensidad. El micrófono utilizado fue ubicado a $10 \mathrm{~cm}$ de la cavidad oral de cada participante y la grabación obtenida fue guardada para, posteriormente, analizar con el software PRAAT los parámetros relacionados con la perturbación de frecuencia, intensidad y ruido; el tiempo máximo fonatorio y el inicio o ataque vocal. Para llevar a cabo las mediciones de los parámetros de perturbación, se utilizaron los 3 segundos centrales de la emisión; para la estimación del tiempo máximo fonatorio se trabajó con la totalidad de lo emitido por la participante. Por último, para la obtención del ataque vocal se utilizó el segmento correspondiente a los 2 segundos iniciales de la emisión. La intensidad fue evaluada gracias al uso de un sonómetro ubicado a $15 \mathrm{~cm}$ de la cavidad oral de cada participante mientras realizaba la /a/ prolongada. Paralelamente, mientras se realizaba esta tarea, uno de los investigadores analizó la voz de cada sujeto según la escala RASATI.

Tanto la grabación de la voz, como la obtención de la intensidad mientras se ejecutaba la emisión de la /a/ prolongada, corresponde a la evaluación inicial de las participantes. 
A continuación, se realizó la tarea de sobrecarga vocal. Cada sujeto debió leer el libro «Paula»por un tiempo de 40 minutos. La intensidad utilizada fluctuó entre los 75 a $85 \mathrm{~dB}$. Para asegurarse que la intensidad requerida se cumpliera, se utilizó un sonómetro a $20 \mathrm{~cm}$ de la cavidad oral de la participante, lo que permitió que uno de los investigadores pudiera monitorear el volumen utilizado. En caso que la voz se emitiera con menos intensidad, se solicitaba de forma verbal y gestual su regulación.

Al terminar la prueba, se le preguntó a cada participante si durante la lectura sintió dolor o sensación de tensión en la región laríngea o faríngea. Esto fue consignado en una hoja que incluía sus datos personales.

Posteriormente, se repitió la evaluación inicial a cada participante con la finalidad de obtener los cambios acústicos producidos por la sobrecarga.

A continuación se llevó a cabo una laringoscopía con luz directa para observar la coloración cordal y perilaríngea y la configuración glótica. Esta última se observó gracias la emisión de una /e/ o /i/ en un tono e intensidad habitual, mientras que la coloración se evaluó durante la respiración.

Posteriormente, a cada sujeto se le colocaron cuatro electrodos; dos en la región submandibular y dos hacia lateral del cartílago tiroides (figura 1). Con respecto a las prestaciones de la electroterapia, el equipo estaba configurado con una frecuencia de $2 \mathrm{~Hz}$, modo normal continuo - esto para ejercer la misma estimulación de forma constante, lo que es recomendado para tiempos de trabajo como el utilizado en esta investigación (Solak et al., 2009) - y un ancho de pulso de $300 \mu \mathrm{seg}$. La intensidad utilizada fue adaptada según la tolerancia -máxima intensidad aceptada por el sujeto y que no produce dolor o sensaciones físicas desagradables; como picazón, ardor o presión (Lazarou, Kitsios, Lazarou, Sikaras, \& Trampas, 2009)- , de este modo, cada uno de los participantes utilizó distintas magnitudes (tabla 1).

Durante la aplicación de la electroestimulación, cada participante debió permanecer en decúbito supino durante 15 minutos y con la instrucción de mantener reposo vocal absoluto.

Al terminar la aplicación de electroterapia, se limpió el cuello de cada sujeto con alcohol y, además, se le preguntó si seguía sintiendo dolor o tensión (dependiendo de la respuesta inicial). Esta respuesta fue consignada en la misma hoja anteriormente utilizada.

Por último, se repitió la evaluación inicial y la examinación laringoscópica, donde se midieron todos los mismos parámetros anteriormente mencionados.

Tabla 1. Intensidad utilizada por cada participante

\begin{tabular}{llllllllllllllllllllll}
\hline Participante & 1 & 2 & 3 & 4 & 5 & 6 & 7 & 8 & 9 & 10 & 11 & 12 & 13 & 14 & 15 & 16 & 17 & 18 & 19 & 20 & 21 \\
\hline $\begin{array}{l}\text { Intensidad } \\
(\mathrm{mA})\end{array}$ & 12 & 13 & 13 & 12 & 13 & 8 & 6 & 13 & 14 & 11 & 15 & 10 & 13 & 7 & 9 & 14 & 12 & 15 & 15 & 16 & 16 \\
\hline
\end{tabular}

\section{Resultados}

El análisis estadístico de los resultados obtenidos se llevó a cabo con el software SPSS versión 20, donde se utilizaron estadísticas descriptivas para todas las variables medidas.

Los resultados conseguidos se exponen a continuación según cada una de las etapas que presentó esta investigación.

\subsection{Resultados previos a la tarea de sobrecarga}

Previo a la tarea de sobrecarga o como estado inicial, se pudo observar que los parámetros de perturbación de tono, amplitud y ruido; frecuencia fundamental, intensidad vocal y duración, se encontraban normales. En la tabla 2 se muestran los mínimos, máximos, promedio y desviación estándar de estos parámetros. En cuanto a la evaluación perceptual, se logró evidenciar que las características acústicas de cada participante eran normales. Con respecto al ataque o inicio vocal, este fue predominantemente suave o normal (figura 2).

\subsection{Resultados posteriores a la tarea de sobrecarga (previo a la electroestimulación)}

Posterior a la tarea de sobrecarga, se observó disminución en los valores de Jitter y Shimmer; mientras que HNR, frecuencia fundamental, intensidad vocal y duración aumentaron (tabla 2). Con respecto a la evaluación perceptual, se evidenció que en el 76,1\% $(\mathrm{n}=16)$ la voz adquirió características de tensión, en el $19 \%(\mathrm{n}=4)$ se presentó predominantemente asténica, mientras que en el 4,8\% $(n=1)$ restante permaneció normal. Con respecto al inicio vocal, este se volvió brusco en el 90,5\% ( $n=19)$, mientras que el 9,5\% $(n=2)$ restante lo mantuvo normal (figura 2).

En lo que refiere a los síntomas de fatiga, el 71,4\% $(n=15)$ refirió sentir dolor o tensión laríngea durante la prueba de sobrecarga. De este porcentaje, el 53,3\% $(\mathrm{n}=8)$ presentó solamente tensión, el $20 \%(\mathrm{n}=3)$ exclusivamente dolor y el 26,6\% (n=4) restante experimentó ambos síntomas. 
Con respecto a los parámetros laringoscópicos (figura 3), se pudo observar que el 66,7\% (n=14) presentó mayor compresión supraglótica. En lo que respecta a la coloración, el 90,5\% $(n=19)$ adquirió mayor enrojecimiento de la cubierta cordal, mientras que el 100\% $(\mathrm{n}=21)$ exhibió este mismo hallazgo en la región perilaríngea.

\subsection{Resultados posteriores a la electroterapia}

En lo que respecta a los parámetros acústicos, se observó que Jitter, Shimmer, HNR, frecuencia fundamental, duración e intensidad, mostraron valores cercanos a los iniciales (previo a la sobrecarga). En la tabla 2 se muestran los mínimos, máximos, promedio y desviación estándar de estos parámetros. En lo que refiere al ataque vocal, el 66,7\% $(n=14)$ presentó un inicio suave o normal, mientras que el 33,3\% $(n=7)$ exhibió un inicio brusco (figura 2).

En relación a los síntomas de fatiga, el $86,7 \%(n=13)$ refirió ya no sentir molestias, mientras el $13,3 \%(n=2)$ las siguió experimentando. De este porcentaje, el $50 \%(\mathrm{n}=1)$ mantuvo la sensación de tensión laríngea, mientras que el $50 \%(n=1)$ restante siguió experimentando dolor.

Con relación a los parámetros laringoscópicos (figura 3), en el 19\% $(n=4)$ se mantuvo la compresión supraglótica, en el $81 \%(n=17)$ disminuyó el enrojecimiento perilaríngeo y en el 71,4\% $(n=15)$ decreció el enrojecimiento de la cubierta cordal.

\section{Discusión}

La electroterapia ha sido utilizada previamente para disminuir estados tensionales o síntomas como la odinofonía (de Oliveira et al., 2015). Sin embargo, en esta investigación se muestra su utilidad en la aceleración de la recuperación de los tejidos que previamente fueron sometidos a tareas de sobrecarga.

Tabla 2. Parámetros acústicos evaluados

\begin{tabular}{|c|c|c|c|c|}
\hline \multirow[t]{2}{*}{$\begin{array}{c}\text { Parámetros } \\
\text { evaluados }\end{array}$} & \multicolumn{4}{|c|}{ Inicial (previo a sobrecarga) } \\
\hline & Valor mínimo & Valor máximo & Promedio & Desviación estándar \\
\hline Jitter (\%) & 0.23 & 0.81 & 0.51 & 0.14 \\
\hline Shimmer (\%) & 3.88 & 14.55 & 9.94 & 2.46 \\
\hline HNR (dB) & 6.26 & 13.21 & 8.54 & 1.72 \\
\hline $\begin{array}{l}\text { Frecuencia fun- } \\
\text { damental (Hz) }\end{array}$ & 210 & 261 & 228.71 & 14.80 \\
\hline $\begin{array}{c}\text { Tiempo máximo } \\
\text { fonatorio (s) }\end{array}$ & 7.60 & 20.67 & 13.31 & 3.53 \\
\hline Intensidad (dB) & 52.90 & 68.25 & 62.25 & 3.01 \\
\hline \multirow[t]{2}{*}{$\begin{array}{c}\text { Parámetros } \\
\text { evaluados }\end{array}$} & \multicolumn{4}{|c|}{ Posterior a sobrecarga (previo a electroterapia) } \\
\hline & Valor mínimo & Valor máximo & Promedio & Desviación estándar \\
\hline Jitter (\%) & 0.17 & 0.85 & 0.38 & 0.16 \\
\hline Shimmer (\%) & 2.66 & 9.85 & 5.97 & 2.18 \\
\hline HNR (dB) & 8.97 & 20.72 & 15.39 & 3.12 \\
\hline $\begin{array}{c}\text { Frecuencia fun- } \\
\text { damental (Hz) }\end{array}$ & 212.50 & 316.50 & 251.52 & 24.89 \\
\hline $\begin{array}{c}\text { Tiempo máximo } \\
\text { fonatorio (s) }\end{array}$ & 8.60 & 20.24 & 16.19 & 4.02 \\
\hline Intensidad (dB) & 60.97 & 71.92 & 67.35 & 3.05 \\
\hline \multirow[t]{2}{*}{$\begin{array}{c}\text { Parámetros } \\
\text { evaluados }\end{array}$} & \multicolumn{4}{|c|}{ Posterior a electroterapia } \\
\hline & Valor mínimo & Valor máximo & Promedio & Desviación estándar \\
\hline Jitter (\%) & 0.25 & 0.70 & 0.41 & 0.95 \\
\hline
\end{tabular}




\begin{tabular}{ccccc}
\hline Shimmer (\%) & 2.40 & 11.83 & 7.25 & 2.57 \\
\hline HNR (dB.) & 7.64 & 18.19 & 11.97 & 2.74 \\
\hline $\begin{array}{c}\text { Frecuencia fun- } \\
\text { damental (Hz.) }\end{array}$ & 207.50 & 281.30 & 232.55 & 19.31 \\
\hline $\begin{array}{c}\text { Tiempo máximo } \\
\text { fonatorio (s.) }\end{array}$ & 8.52 & 22.94 & 14.65 & 3.62 \\
\hline \begin{tabular}{c} 
Intensidad (dB.) \\
\hline
\end{tabular} & 54.97 & 70.31 & 63.16 & 3.38 \\
\hline
\end{tabular}

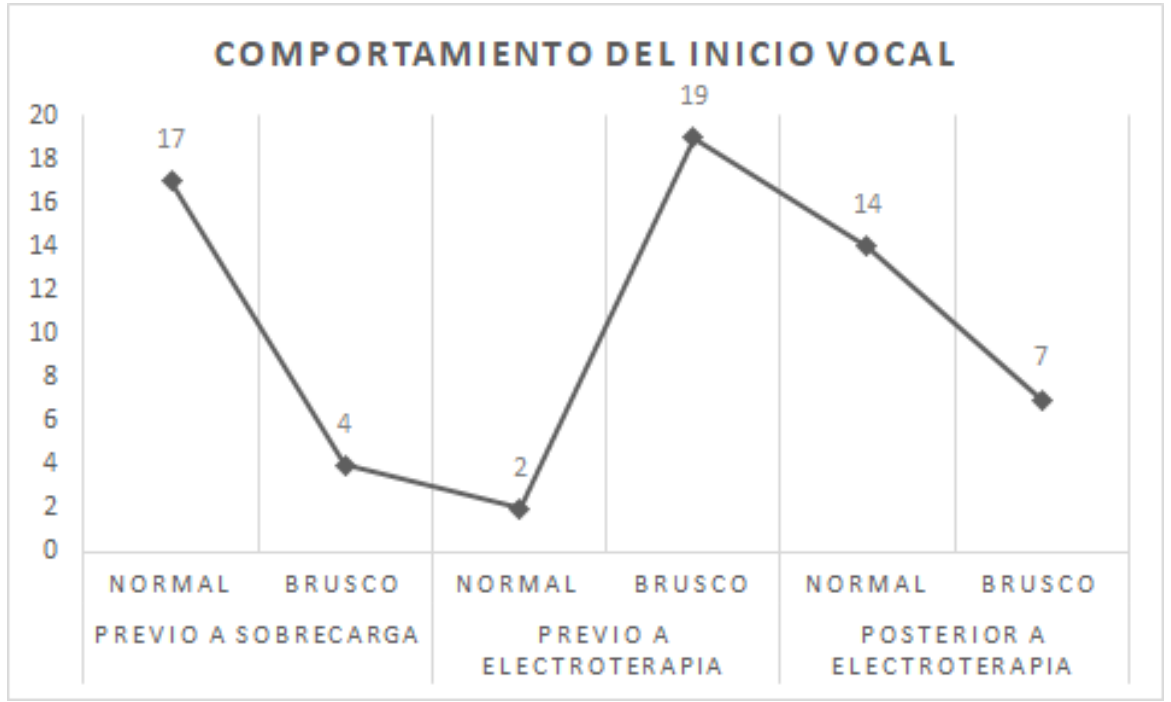

Figura 2. Evolución del inicio o ataque vocal en la muestra estudiada.

En este estudio se ha utilizado una configuración de electroterapia que no se ha indicado anteriormente en las investigaciones relacionadas con la terapéutica vocal. Dentro de esto destaca el uso de frecuencias bajas (TENS de tipo acupuntura) y la aplicación de cuatro electrodos.

El uso de TENS de tipo acupuntura se debe principalmente al efecto de recuperación posterior a la sobrecarga que ejerce en otras partes del cuerpo (Jun et al., 2015), los que a su vez, no se han documentado para corrientes de mayor frecuencia. Mientras que la utilización de cuatro electrodos recae en las repercusiones perilaríngeas que ofrece la sobrecarga vocal (Fuentes, Biermann, \& Portillo, 2019). Previamente, se ha observado que durante la fonación se pueden sobreutilizar los músculos supra e infrahioideos, sus tendones y hasta la red ligamentosa ubicada entre hioides y laringe (Aydil et al., 2007; O’Rourke et al., 2014). La búsqueda de más electrodos implica una mayor área de estimulación (Starkey, 2013) y por consiguiente, la posibilidad de abarcar a aquellos tejidos que estuvieron siendo utilizados para producir la articulación, resonancia y fonación durante la prueba de sobrecarga vocal (Stern et al., 2013).

El tiempo de aplicación correspondió a una elección proveniente de los tiempos naturales de recuperación que tienen los tejidos posterior a la sobrecarga. Se ha considerado que en sobrecargas similares a las utilizadas en este estudio, la recuperación musculoesquelética va desde los 60 minutos hasta las 24 horas (Whitling, Rydell, \& Lyberg Åhlander, 2015), por lo que la presencia de resultados beneficiosos expresan con claridad el potencial recuperativo que tiene la electroestimulación de tipo acupuntura sobre los tejidos implicados en la fonación.

Según lo observado, las repercusiones de la electroterpia de baja frecuencia se pueden manifestar en la cubierta cordal, músculos laríngeos intrínsecos y en la región perilaríngea.

\subsection{Efectos sobre la región perilaríngea}

La región periláringea se compone por una serie de tejidos blandos que incluyen a tendones, ligamentos y músculos (Sonoda, Naohiro \& Tamatsu, 2008). Cada uno de estos elementos es sensible a la sobrecarga o al sobreuso que ofrecen agentes mecánicos como la fonación (O'Rourke et al., 2014). Cuando la sobrecarga es intensa o repetitiva, estos tejidos expresan una serie de signos y síntomas vocales, los que incluyen a la odinofonía (Jung et al., 2017) y, en algunos casos, a la aparición de compresión supraglótica (Khoddami, Nakhostin Ansari, Izadi, \& Talebian, 2013). Se ha teorizado que la sobreactividad del tirohioideo incide directamente en la aparición de compresión medial (Van Houtte, Van Lierde, \& Claeys, 2011). Paralelamente, también se ha indicado que este tipo de configuraciones surgi- 
rían como adaptación al uso constante de la voz en frecuencias y tonos superiores a los acostumbrados, y que, por lo tanto, su presencia hablaría de una adaptación de los tejidos a la sobrecarga (Mayerhoff et al., 2014). En la muestra estudiada, la aparición de compresión supraglótica y de síntomas perilaríngeos posterior a la prueba de lectura fueron hechos frecuentes y repetitivos.

El uso de la electroestimulación tiene repercusiones positivas sobre tejidos como los existentes en la región perilaríngea. Se ha indicado que con su aplicación se mejora la vascularización (Machado, Santana, Tacani, \& Liebano, 2012) y se disminuye la rigidez muscular (Karasuno et al., 2016). De esta forma, y considerando lo explicado anteriormente, la disminución en la compresión supraglótica y de la sensación de dolor o tensión, son efectos atribuidos al uso de este agente sobre la región perilaríngea.
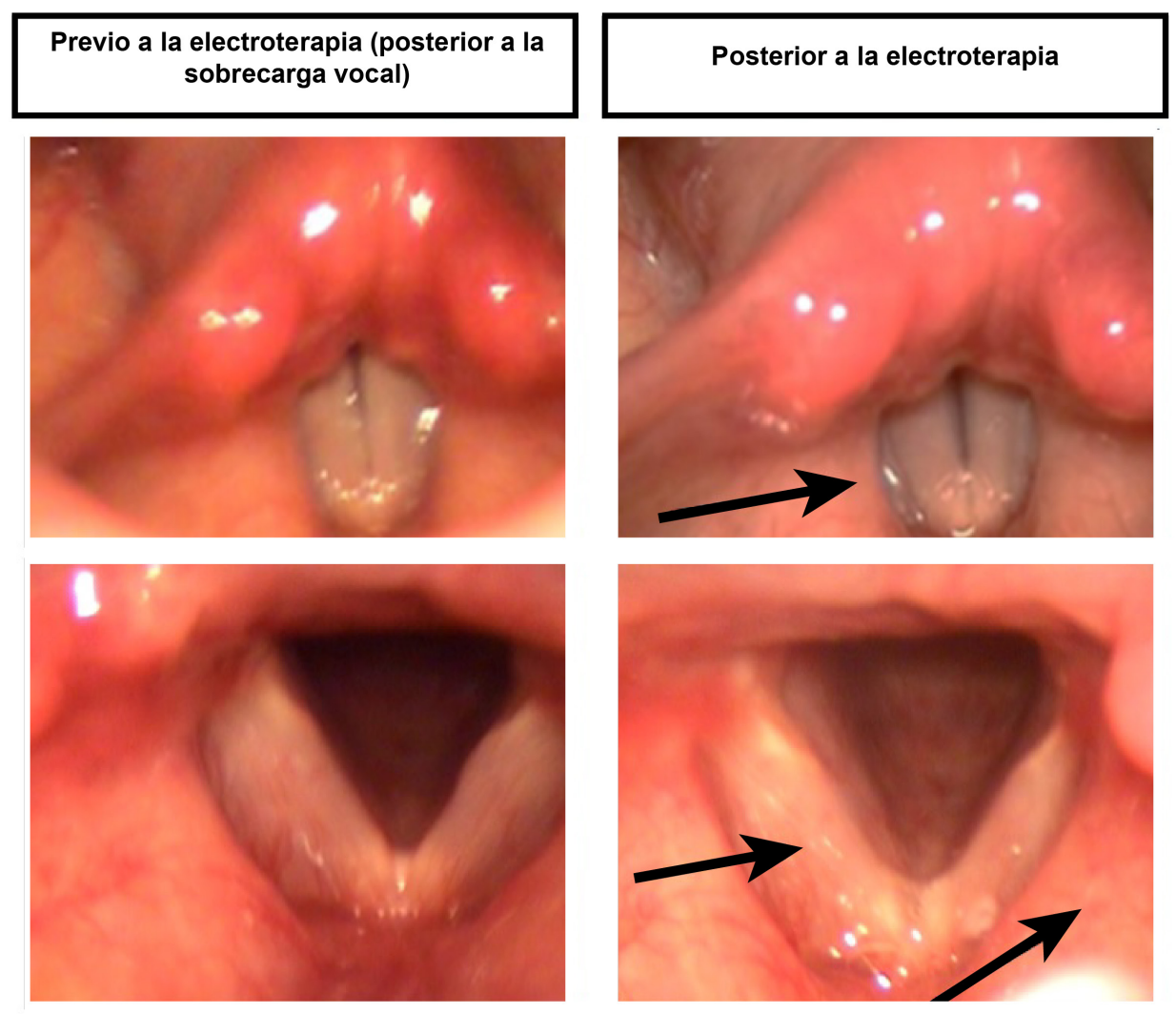

Figura 3. Cambios laringoscópicos observados antes y después de la electroterapia. En superior se observa la reducción de la compresión supraglótica (flecha negra), mientras que en inferior se evidencia la disminución del enrojecimiento cordal y perilaríngeo.

Las repercusiones expresadas sobre los tejidos perilaríngeos ofrecen una importante ayuda en la intercepción de su injuria. Gracias a su aplicación se evitarían patrones musculoesqueléticos propios de la sobrecarga, como la mantención durante el reposo de la posición elevada de la laringe y hioides (Lowell, Kelley, Colton, Smith, \& Portnoy, 2012) o el aumento de la sensibilidad hioidea que favorece la aparición de odinofonía (O'Rourke et al., 2014). A pesar de lo anterior, se ha visto que la corriente aplicada en esta investigación no entrega los mejores efectos sobre la recuperación muscular (Neric, Beam, Brown, \& Wiersma, 2009).

\subsection{Efectos sobre los músculos intrínsecos}

El control de la intensidad y de la frecuencia fundamental depende principalmente del delicado mecanismo que existe en los músculos intrínsecos de la laringe (Zhang, 2016). Cuando la voz es sometida a constante sobrecarga, estos músculos se adaptan y adquieren características que permiten al sujeto mantener las particularidades fonatorias propias de la misma sobrecarga (Remacle, Morsomme, \& Finck, 2014). Esto incluye al aumento de la intensidad habitual, incremento del tiempo máximo fonatorio (Fujiki \& Sivasankar, 2017), y disminución del jitter y shimmer. Sin embargo, también se observan efectos que indican la fatiga metabólica secundaria a la sobrecarga, donde sobresale el aumento de HNR (Fujiki \& Sivasankar, 2017). Todas estas consecuencias fueron observadas en la muestra estudiada.

Adicionalmente, los efectos sobre la tensión muscular intrínseca que ofrece la sobrecarga también han sido observados en esta investigación. La aparición de ataque vocal duro es la principal expresión de este fenómeno, específicamente, la sobreactividad del cricoaritenoideo lateral, de la rama vocalis del tiroaritenoideo y del interaritenoideo (Hirose \& Gay, 1973). 
En esta investigación se observaron beneficios que implican la disminución de la hiperactividad muscular intrínseca, los que incluyen a la suavización del inicio vocal. Estos cambios son atribuidos a las características de relajación que entrega la corriente utilizada (Karasuno et al., 2016).

\subsection{Efectos sobre la cubierta cordal}

La cubierta cordal presenta una serie de elementos histológicos, los que le permiten soportar a las diversas fuerzas mecánicas existentes durante la oscilación cordal (Titze, 1994). Cuando la fonación es intensa o repetitiva, esta estructura expresa consecuencias que incluyen a su inflamación (Titze, Svec, \& Popolo, 2003). En esta muestra, el enrojecimiento posterior a la tarea realizada corrobora esta situación. Se ha planteado que la aplicación de electroterapia favorece la reducción de citoquinas proinflamatorias en las áreas donde se aplica y, por consiguiente, facilita la reducción del proceso inflamatorio (do Carmo Almeida et al., 2018). Adicionalmente, también se ha observado que el uso de corriente TENS favorece la proliferación celular y con eso acelera procesos propios de la recuperación, los que incluyen tiempos de inflamación más cortos (Thakral et al., 2013). Procesos como los expresados, darían explicación a la disminución del enrojecimiento de la cubierta cordal y del tejido perilaríngeo que se evidenció posterior a la aplicación de electroterapia.

Anatómicamente, las cuerdas vocales se ubican bajo una importante barrera para la electroestimulación, y esta es la capa de tejido adiposo del cuello. Se ha estimado que este tejido es capaz de dificultar la transmisión del impulso eléctrico (Doheny, Caulfield, Minogue, \& Lowery, 2008), por lo que los efectos de la electroestimulación en estructuras profundas como las propias de los pliegues vocales, se verán condicionadas por el grosor o cantidad de tejido adiposo del cuello.

A pesar de todo lo explicado a lo largo de esta discusión, efectos como los mencionados pueden ser atribuidos al tiempo de reposo vocal absoluto que mantuvo la muestra mientras se aplicaba la electroterapia. No obstante, las consecuencias del reposo vocal se han evidenciado previamente y se discuten a continuación.

\subsection{Efectos del reposo vocal posterior a la sobrecarga vocal.}

Posterior a la sobrecarga, la cubierta cordal suele exhibir modificaciones que indican su inflamación (Fujiki \& Sivasankar, 2017). El enrojecimiento posterior a la tarea realizada corrobora esta situación. A su vez, existen cambios que indican la sobreactivación del mecanismo tiroaritenoideo, lo que ocasiona el aumento de intensidad, frecuencia fundamental o tiempo máximo fonatorio (Whitling et al., 2017). Adicionalmente, existen adaptaciones supraglóticas que buscan la obtención de mayores niveles de intensidad o de frecuencia fundamental (según lo requerido por la tarea) (Samuel, Mahalingam, Balasubramaniyam, Boominathan, \& Arunachalam, 2011).

Se ha planteado que el reposo puede funcionar como factor de recuperación de todos estos efectos. Sin embargo, se han visto cambios positivos en tiempo superiores a los aplicados a la muestra estudiada. En tiempos de sobrecarga de 45 minutos con intensidades superiores a $\operatorname{los} 75 \mathrm{~dB}$, se ha observado una recuperación vocal completa a las 24 horas posterior a la tarea (Samuel et al., 2011). En otro trabajo se mantuvo a profesores leyendo durante dos horas a una intensidad similar a la usada en clases. Se evidenciaron dos curvas de recuperación, una relacionada con el mecanismo tiroaritenoideo y la otra con la lámina propia. Para la primera, se necesitó de 4 a 6 horas para que desaparecieran los efectos del sobreuso de los músculos intrínsecos de las cuerdas vocales. Mientras que para la segunda se necesitó de 12 a 18 horas de reposo para eliminar las molestias relacionadas con la fatiga de la lámina propia (Hunter \& Titze, 2009). Otra investigación utilizó dos horas de fonación en un rango de intensidad entre 75 a 85 dB, se observó que el mínimo umbral fonatorio y el esfuerzo fonatorio percibido comenzaron su restauración a las dos horas posterior a la tarea, recuperándose en su totalidad a las 72 horas (Chang \& Karnell, 2004). También se ha indicado que en tiempo cortos, menores a una hora inclusive, pueden servir para la recuperación neuromuscular; dependiendo de la complejidad de la tarea ejecutada (McCabe \& Titze, 2002).

De esta manera, los efectos positivos del reposo se expresarían en tiempos superiores a los utilizados por la muestra estudiada, por lo que las consecuencias presentadas y discutidas si pueden atribuirse a la electroestimulación. No obstante, es posible que sus principales efectos estén vinculados hacia la hiperactividad perilaríngea, disminución de la tensión laríngea o dolor y desinflamación de los tejidos sobreutilizados. El sobreuso tiroaritenoideo, tal como se ha evidenciado en la literatura, se beneficiaría principalmente por la aplicación del reposo vocal.

La sobrecarga es un fenómeno diario y común en los profesionales de la voz, el uso de estrategias que permitan su intercepción y con eso, la prevención del daño funcional, es primordial como objetivo terapéutico. En investigaciones previas se ha estimado que el uso de estrés tensil favorece la desinflamación de la cubierta cordal (Verdolini Abbott et al., 2012). Si estos efectos se suman a los entregados por la electroterapia, se formará una importante estrategia de prevención cuya finalidad será que los profesionales de la voz puedan evitar los problemas por carga acumulada.

El uso de la electroterapia como forma de recuperación posterior a la sobrecarga vocal es novedoso, por lo que se recomienda profundizar en su estudio y utilidad. De esta forma, se recomienda crear nuevos diseños de investigación, los que incluyan a otras poblaciones de estudio, comparaciones directas con el reposo vocal y con otros agentes físicos de rehabilitación. Adicionalmente, se sugiere profundizar en este tipo de investigaciones con otras configura- 
ciones de electroestimulación, tales como, mayor o menor frecuencia, utilización de pares de electrodos que conduzcan frecuencias específicas (con esto se evita el enviar el mismo tipo de corriente en todos los canales) y diferentes tiempos de estimulación. Por último, es necesario utilizar muestras de mayor tamaño a las de esta investigación para obtener resultados más representativos.

\section{Conclusiones}

La electroestimulación de tipo acupuntura ha mostrado efectos beneficiosos en la recuperación de los tejidos utilizados durante la sobrecarga vocal. Sus principales efectos se relacionan con la relajación o disminución de la rigidez perilaríngea, lo que repercute directamente en la sensación de tensión y dolor. Además, favorece la desinflamación de la cubierta cordal y beneficia la relajación intrínseca laríngea, disminuyendo así, la incidencia de ataque vocal duro.

\section{Bibliografía}

Aydil, U., Ekinci, Ö., Köybaşioğlu, A., \& Kizil, Y. (2007). Hyoid bone insertion tendinitis: clinicopathologic correlation. European Archives of Oto-Rhino-Laryngology, 264(5), 557-560. https://doi.org/10.1007/s00405-006-0220-x

Babault, N., Cometti, C., Maffiuletti, N. A., \& Deley, G. (2011). Does electrical stimulation enhance post-exercise performance recovery? European Journal of Applied Physiology, 111(10), 2501-2507. https://doi.org/10.1007/s00421-011-2117-7

Bastian, R. W., \& Thomas, J. P. (2016). Do Talkativeness and Vocal Loudness Correlate With Laryngeal Pathology? A Study of the Vocal Overdoer/Underdoer Continuum. Journal of Voice, 30(5), 557-562. https://doi.org/10.1016/j.jvoice.2015.06.012

Bisciotti, G. N., \& Eirale, C. (2013). Muscle injuries in sport medicine. (InTech, Ed.). Rijeka.

Cameron, M. H. (2014). Agentes físicos en rehabilitación : de la investigación a la práctica. Elsevier España.

Chang, A., \& Karnell, M. P. (2004). Perceived phonatory effort and phonation threshold pressure across a prolonged voice loading task: A study of vocal fatigue. Journal of Voice, 18(4), 454-466. https://doi.org/10.1016/j.jvoice.2004.01.004

de Oliveira, J., Côrtes, A. C., Alves, K., Cordeiro, N., Santos, J. K. de O., Gama, A. C. C., ... Oliveira, N. F. C. D. (2015). Uso da eletroestimulação na clínica fonoaudiológica: uma revisão integrativa da literatura. Revista CEFAC, 17(5), $1620-1632$. https://doi.org/10.1590/1982-0216201517518114

do Carmo Almeida, T. C., dos Santos Figueiredo, F. W., Barbosa Filho, V. C., de Abreu, L. C., Fonseca, F. L. A., \& Adami, F. (2018). Effects of Transcutaneous Electrical Nerve Stimulation on Proinflammatory Cytokines: Systematic Review and Meta-Analysis. Mediators of Inflammation, 2018, 1-13. https://doi.org/10.1155/2018/1094352

Doheny, E. P., Caulfield, B. M., Minogue, C. M., \& Lowery, M. M. (2008). The effect of subcutaneous fat thickness on the efficacy of transcutaneous electrical stimulation. In 2008 30th Annual International Conference of the IEEE Engineering in Medicine and Biology Society (Vol. 2008, pp. 5684-5687). IEEE. https://doi.org/10.1109/IEMBS.2008.4650504

Esclassan, R., Rumerio, A., Monsarrat, P., Combadazou, J. C., Champion, J., Destruhaut, F., \& Ghrenassia, C. (2017). Optimal duration of ultra low frequency-transcutaneous electrical nerve stimulation (ULF-TENS) therapy for muscular relaxation in neuromuscular occlusion: A preliminary clinical study. CRANIO®, 35(3), 175-179. https://doi.org/10.1080/08869634.2016 .1171479

Fowler, L. P., Awan, S. N., Gorham-Rowan, M., \& Morris, R. (2011). Investigation of Fatigue, Delayed-Onset Muscle Soreness, and Spectral-Based Cepstral Measurements in Healthy Speakers after Neuromuscular Electrical Stimulation. Annals of Otology, Rhinology \& Laryngology, 120(10), 641-650. https://doi.org/10.1177/000348941112001003

Fuentes, C. (2018). La carga vocal. Definición, fonotrauma y prescripción. (Brujas, Ed.) (Primera).

Fuentes, C., Biermann, C., \& Portillo, J. (2019). Consecuencias de la sobrecarga vocal en el umbral doloroso de los tejidos perilaríngeos. Revista de Logopedia, Foniatría y Audiología, En prensa.

Fujiki, R. B., \& Sivasankar, M. P. (2017). A Review of Vocal Loading Tasks in the Voice Literature. Journal of Voice, 31 (3), 388. e33-388.e39.

Guirro, R. R. de J., Bigaton, D. R., Silvério, K. C. A., Berni, K. C. dos S., Distéfano, G., Santos, F. L. dos, \& Forti, F. (2008). Transcutaneous electrical nerve stimulation in dysphonic women. Pro-Fono : Revista de Atualizacao Cientifica, 20(3), 189195.

Hansson, P., \& Ekblom, A. (1983). Transcutaneous electrical nerve stimulation (TENS) as compared to placebo TENS for the relief of acute oro-facial pain. Pain, 15(1), 157-165. https://doi.org/10.1016/0304-3959(83)90015-5

Hirose, H., \& Gay, T. (1973). Laryngeal Control in Vocal Attack. Folia Phoniatrica et Logopaedica, 25(3), 203-213. https://doi. org/10.1159/000263688

Hunter, E. J., \& Titze, I. R. (2009). Quantifying vocal fatigue recovery: dynamic vocal recovery trajectories after a vocal loading exercise. The Annals of Otology, Rhinology, and Laryngology, 118(6), 449-460.

Jun, M.-H., Kim, Y.-M., \& Kim, J. U. (2015). Modern acupuncture-like stimulation methods: a literature review. Integrative Medicine Research, 4(4), 195-219. https://doi.org/10.1016/j.imr.2015.09.005

Jung, S. Y., Park, H. J. H. S., Bae, H., Yoo, J. H., Park, H. J. H. S., Park, K. D., .. Chung, S. M. (2017). Laryngeal myofascial pain syndrome as a new diagnostic entity of dysphonia. Auris Nasus Larynx, 44(2), 182-187. https://doi.org/10.1016/j. anl.2016.05.001 
Kang, D. H., Jeon, J. K., \& Lee, J. H. (2015). Effects of low-frequency electrical stimulation on cumulative fatigue and muscle tone of the erector spinae. Journal of Physical Therapy Science, 27(1), 105-108. https://doi.org/10.1589/jpts.27.105

Karasuno, H., Ogihara, H., Morishita, K., Yokoi, Y., Fujiwara, T., Ogoma, Y., \& Abe, K. (2016). The combined effects of transcutaneous electrical nerve stimulation (TENS) and stretching on muscle hardness and pressure pain threshold. Journal of Physical Therapy Science, 28(4), 1124-1130. https://doi.org/10.1589/jpts.28.1124

Khoddami, S. M., Nakhostin Ansari, N., Izadi, F., \& Talebian Moghadam, S. (2013). The assessment methods of laryngeal muscle activity in muscle tension dysphonia: a review. The Scientific World Journal, 2013, 507397. https://doi. org/10.1155/2013/507397

Lazarou, L., Kitsios, A., Lazarou, I., Sikaras, E., \& Trampas, A. (2009). Effects of intensity of transcutaneous electrical nerve stimulation (TENS) on pressure pain threshold and blood pressure in healthy humans: A randomized, double-blind, placebo-controlled trial. Clinical Journal of Pain, 25(9), 773-780. https://doi.org/10.1097/AJP.0b013e3181a7ece3

Lowell, S. Y., Kelley, R. T., Colton, R. H., Smith, P. B., \& Portnoy, J. E. (2012). Position of the Hyoid and Larynx in People With Muscle Tension Dysphonia. Laryngoscope, 122(2), 370-377. https://doi.org/10.1002/lary.22482

Machado, A. F. P., Santana, E. F., Tacani, P. M., \& Liebano, R. E. (2012). The effects of transcutaneous electrical nerve stimulation on tissue repair: A literature review. The Canadian Journal of Plastic Surgery = Journal Canadien de Chirurgie Plastique, 20(4), 237-240.

Mansuri, B., Torabinezhad, F., Jamshidi, A. A., Dabirmoghadam, P., Vasaghi-Gharamaleki, B., \& Ghelichi, L. (2019). Application of High-Frequency Transcutaneous Electrical Nerve Stimulation in Muscle Tension Dysphonia Patients With the Pain Complaint: The Immediate Effect. Journal of Voice. https://doi.org/10.1016/j.jvoice.2019.02.009

Mayerhoff, R. M., Guzman, M., Jackson-Menaldi, C., Munoz, D., Dowdall, J., Maki, A., ... Rubin, A. D. (2014). Analysis of supraglottic activity during vocalization in healthy singers. The Laryngoscope, 124(2), 504-509. https://doi.org/10.1002/ lary. 24310

McCabe, D. J., \& Titze, I. R. (2002). Chant Therapy For Treating Vocal Fatigue Among Public School Teachers. American Journal of Speech-Language Pathology, 11(4), 356-369. https://doi.org/10.1044/1058-0360(2002/040)

Mummolo, S., Nota, A., Tecco, S., Caruso, S., Marchetti, E., Marzo, G., \& Cutilli, T. (2018). Ultra-low-frequency transcutaneous electric nerve stimulation (ULF-TENS) in subjects with craniofacial pain: A retrospective study. CRANIO ${ }^{\circledR}, 1-6$. https://doi. org/10.1080/08869634.2018.1526849

Neric, F. B., Beam, W. C., Brown, L. E., \& Wiersma, L. D. (2009). Comparison of Swim Recovery and Muscle Stimulation on Lactate Removal After Sprint Swimming. Journal of Strength and Conditioning Research, 23(9), 2560-2567. https://doi. org/10.1519/JSC.0b013e3181bc1b7a

O’Rourke, C., Attique, S., Rehman, A. U., Saunders, J., \& Fenton, J. E. (2014). Hyoid Bone Tenderness as a Clinical Indicator of Laryngeal Pathology. Journal of Voice, 28(6), 835-837. https://doi.org/10.1016/j.jvoice.2014.02.012

Peterson, M. D., Pistilli, E., Haff, G. G., Hoffman, E. P., \& Gordon, P. M. (2011). Progression of volume load and muscular adaptation during resistance exercise. European Journal of Applied Physiology, 111(6), 1063-1071. https://doi.org/10.1007/ s00421-010-1735-9

Petrofsky, J. S., Khowailed, I. A., Lee, H., Berk, L., Bains, G. S., Akerkar, S., ... Laymon, M. S. (2015). Cold Vs. Heat After Exercise-Is There a Clear Winner for Muscle Soreness. Journal of Strength and Conditioning Research, 29(11), $3245-3252$. https://doi.org/10.1519/JSC.0000000000001127

Phillips, E. M., \& Kennedy, M. A. (2012). The Exercise Prescription: A Tool to Improve Physical Activity. PM\&R, 4(11), 818825. https://doi.org/10.1016/j.pmrj.2012.09.582

Remacle, A., Morsomme, D., \& Finck, C. (2014). Comparison of Vocal Loading Parameters in Kindergarten and Elementary School Teachers. Journal of Speech, Language, and Hearing Research, 57(2), 406-415. https://doi.org/10.1044/2013 JSLHR-S-12-0351

Rousseau, B., Cohen, S. M., Zeller, A. S., Scearce, L., Tritter, A. G., \& Garrett, C. G. (2011). Compliance and Quality of Life in Patients on Prescribed Voice Rest. Otolaryngology-Head and Neck Surgery, 144(1), 104-107. https://doi. org/10.1177/0194599810390465

Samuel, J., Mahalingam, S., Balasubramaniyam, S., Boominathan, P., \& Arunachalam, R. (2011). Stroboscopic and Multiparametric Acoustic Analysis of Voice after Vocal Loading Task. International Journal of Phonosurgery and Laryngology, 1(2), 47-51. https://doi.org/10.5005/jp-journals-10023-1012

Solak, O., Emmiler, M., Ela, Y., Dündar, U., Koçoiullari, C. U., Eren, N., ... Kavuncu, V. (2009). Comparison of continuous and intermittent transcutaneous electrical nerve stimulation in postoperative pain management after coronary artery bypass grafting: a randomized, placebo-controlled prospective study. The Heart Surgery Forum, 12(5), E266-71. https://doi.org/10.1532/ HSF98.20081139

Sonoda, Naohiro; Tamatsu, Y. (2008). Observation on the attachment of muscles onto the hyoid bone in human adults, 85(3), 79-90. https://doi.org/10.2535/ofaj.85.79

Starkey, C. (2013). Therapeutic modalities. (F.A. Davis Company, Ed.) (Fourth).

Stern, N., Jackson-Menaldi, C., \& Rubin, A. D. (2013). Hyoid Bone Syndrome: A Retrospective Review of 84 Patients Treated with Triamcinolone Acetonide Injections. Annals of Otology, Rhinology \& Laryngology, 122(3), 159-162. https://doi. org/10.1177/000348941312200303

Thakral, G., Lafontaine, J., Najafi, B., Talal, T. K., Kim, P., \& Lavery, L. A. (2013). Electrical stimulation to accelerate wound healing. Diabetic Foot \& Ankle, 4. https://doi.org/10.3402/dfa.v4i0.22081 
Titze, I. R. (1994). Mechanical stress in phonation. Journal of Voice, 8(2), 99-105. https://doi.org/10.1016/S0892-1997(05)803029

Titze, I. R., Svec, J. G., \& Popolo, P. S. (2003). Vocal dose measures: quantifying accumulated vibration exposure in vocal fold tissues. Journal of Speech, Language, and Hearing Research : JSLHR, 46(4), 919-932.

Tousignant-Laflamme, Y., Brochu, M., Dupuis-Michaud, C., Pagé, C., Popovic, D., \& Simard, M.-E. (2013). Duration of Analgesia Induced by Acupuncture-Like TENS on Experimental Heat Pain. ISRN Pain, 2013, 1-6. https://doi.org/10.1155/2013/792383

Van Houtte, E., Van Lierde, K., \& Claeys, S. (2011). Pathophysiology and Treatment of Muscle Tension Dysphonia: A Review of the Current Knowledge. Journal of Voice, 25(2), 202-207. https://doi.org/10.1016/j.jvoice.2009.10.009

Verdolini Abbott, K., Li, N., Branski, R., Rosen, C., Grillo, E., Steinhauer, K., \& Hebda, P. (2012). Vocal exercise may attenuate acute vocal fold inflammation. Journal of Voice : Official Journal of the Voice Foundation, 26(6), 814.e1-13. https://doi. org/10.1016/j.jvoice.2012.03.008

Whitling, S., Lyberg-Åhlander, V., \& Rydell, R. (2017). Recovery From Heavy Vocal Loading in Women With Different Degrees of Functional Voice Problems. Journal of Voice : Official Journal of the Voice Foundation, 31(5), 645.e1-645.e14. https://doi. org/10.1016/j.jvoice.2016.12.012

Whitling, S., Rydell, R., \& Lyberg Åhlander, V. (2015). Design of a Clinical Vocal Loading Test With Long-Time Measurement of Voice. Journal of Voice, 29(2), 261.e13-261.e27. https://doi.org/10.1016/j.jvoice.2014.07.012

Zhang, Z. (2016). Mechanics of human voice production and control. The Journal of the Acoustical Society of America, 140(4), 2614. https://doi.org/10.1121/1.4964509 